\title{
Dynamic Correlation between Whisking and Breathing Rhythms in Mice
}

\author{
Ying Cao (曹荣), ${ }^{1 \star}$ Snigdha Roy, ${ }^{1 \star}$ Robert N. S. Sachdev, ${ }^{2}$ and Detlef H. Heck ${ }^{1}$ \\ ${ }^{1}$ Department Anatomy and Neurobiology, University of Tennessee Health Science Center, Memphis, Tennessee 38163, and ${ }^{2}$ Department of Neurobiology, \\ Yale University School of Medicine, New Haven Connecticut 06520
}

Sniffing, a high-frequency, highly rhythmic inhalation and exhalation of air through the nose, plays an important role in rodent olfaction. Similarly, whisking, the active rhythmic movement of whiskers, plays an important role in rodent tactile sensation. Rodents whisk and sniff during exploratory behavior to sample odorants and surfaces. Whisking is thought to be coordinated with sniffing and normal respiratory behavior, but the precise temporal relationships between these movements are not known. Here, using direct measurements of whisking and respiratory movements, we examined the strength and temporal dynamics of the correlation between large-amplitude whisker movements and respiratory rhythm in mice. Whisking movements were detected using an optical sensor, and respiration was monitored with a thermistor placed close to the nostril. Our measurements revealed that breathing and whisking movements were significantly correlated only when the whisking rhythm was $<5 \mathrm{~Hz}$. Only a fraction $(\sim 13 \%)$ of all large-amplitude whisker movements occurred during episodes of high-frequency $(>5 \mathrm{~Hz})$ respiration typically associated with sniffing. Our results show that that the rhythms of respiratory and whisking movements are correlated only during low-frequency whisking and respiration. High-frequency whisking and sniffing behaviors are not correlated. We conclude that whisking and respiratory rhythms are generated by independent patterngenerating mechanisms.

\section{Introduction}

Exploratory behavior in rats and mice is characterized by highfrequency $(>5 \mathrm{~Hz})$ respiratory or sniffing and whisking behaviors for olfactory and tactile sampling of the environment. The frequency of respiratory and whisking movements, are highly variable depending on the behavioral context but have largely overlapping frequencies within a range of 1-20 Hz (Carvell and Simons, 1996; Voigts et al., 2008; Wesson et al., 2008). Though sniffing and whisking are goal directed, neither requires an intact cortex (Welker, 1964; Hutson and Masterton, 1986; Carvell and Simons, 1996), implying that brainstem circuitry by itself can generate these rhythms. The question arises whether these two rhythmic behaviors that occur within the same frequency range, and the same behavioral context are coordinated with each other or are completely independent.

Though anecdotal, earlier work in rats by Welker, described a temporal relationship between these behaviors. He observed that

\footnotetext{
Received Aug. 27, 2011; revised 0ct. 27, 2011; accepted Dec. 12, 2011.

Author contributions: R.N.S.S. and D.H.H. designed research; Y.C. and S.R. performed research; Y.C., S.R., and D.H.H. analyzed data; Y.C., R.N.S.S., and D.H.H. wrote the paper.

This work was supported by grants from the National Institutes of Health to D.H.H. (R01NS060887 and R01NS067201). The content of this publication is solely the responsibility of the authors and does not necessarily represent the official views of the NIH. We thank Michael Nguyen for excellent custom mechanical designs and Shuhua Qi for outstanding technical assistance. We thank Ben Sagot and Jeremy Edgerton for help with timeresolved correlation analysis.

*Y.C. and S.R. contributed equally.

The authors declare no competing financial interests.

Correspondence should be addressed to Dr. DetlefH. Heck, Department of Anatomy and Neurobiology, University of Tennessee Health Science Center, 855 Monroe Avenue, Room 409, Memphis, TN 38163.E-mail: dheck@uthsc.edu. DOI:10.1523/JNEUROSCI.4395-11.2012

Copyright $\odot 2012$ the authors $\quad 0270-6474 / 12 / 321653-07 \$ 15.00 / 0$
}

when the nose contacted an object, the vibrissae were fully protracted, the tip of the nose was fully retracted, and the peak of inhalation occurred at this moment, when the vibrissae and nose were out of phase (Welker, 1964). Similarly, when the animal withdrew from the object, the vibrissae retracted, the tip of the nose protracted, and the animal exhaled. These observations suggest that in a limited context of the nose touching an object, there exists a very definite relationship between these behaviors. But rodents sniff without touching their nose against objects. Later work, has linked whisking and sniffing together in different contexts, for example during odor discrimination (Kepecs et al., 2007), or during theta synchronized sniffing behaviors (Semba and Komisaruk, 1984). Here we investigated the correlation between large-amplitude in-air whisker movements, as they are typically observed during free exploration and respiratory movements in mice. Our results show that whisking and respiratory movements are correlated with each other only during lowfrequency $(<5 \mathrm{~Hz})$ whisking and breathing rhythms. At higher frequencies of whisking the respiratory and whisking movements were uncorrelated. High-frequency respiration or sniffing only rarely coincided with whisking movements. The vast majority of whisking movements $(\sim 87 \%)$ occurred during low-frequency $(<5 \mathrm{~Hz})$ respiratory movements.

\section{Materials and Methods}

Experiments were performed on adult C57BL/6J (B6) mice of either sex (18-25 g body weight). Mice were housed within a breeding colony with $12 \mathrm{~h}$ light/dark cycles in standard cages with ad libitum access to food and water. All animal experimental procedures adhered to guidelines approved by the University of Tennessee Health Science Center Animal 
Care and Use Committee. Principles of laboratory animal care (NIH Publication No. 86-23, rev. 1996) were followed.

Surgery. The mice were anesthetized initially with $3 \%$ isoflurane in Oxygen in an incubation chamber. Anesthesia was maintained with $1-2 \%$ isoflurane during surgery using an Ohio isoflurane vaporizer (Highland Medical Equipment). The depth of anesthesia was adjusted until the mice failed to show a reflex withdrawal of the hindpaw to a strong pinch. Rectal temperature was maintained at $37-38^{\circ} \mathrm{C}$ with a servo-controlled heat blanket (FHC, Inc.). A metal headpost was mounted relative to bregma and secured into place with dental acrylic, as described in detail previously (Bryant et al., 2009). Mice were injected with a $5 \mathrm{mg} / \mathrm{kg}$ dose of the analgesic Torbugesic (Fort Dodge) to alleviate pain and $0.5 \mathrm{ml}$ of lactated Ringer solution (s.c.) as a fluid supplement twice for the first $24 \mathrm{~h}$ after the surgery. After a 3 - to 4 -d recovery period, mice were adapted to the head-fixed situation during 2 sessions of head fixation of $15 \mathrm{~min}$.

Monitoring of respiratory and whisking behavior. All experiments were performed during the light cycle. Mice were immobilized by fixing the surgically implanted headposts to a stationary metal clamp, and the body was loosely restrained using a hard-plastic cover (Bryant et al., 2009). On the left side of the face all but the $\mathrm{C} 4$ or the $\mathrm{C} 3$ whisker were cut. A light-beam sensor (Coulbourn Instruments) was placed beside the mouse's head at the level of the nose so that the whisker would move through the light beam during large-amplitude protraction movements. The whisker moving through the beam resulted in a fast two-phase descending-ascending potential for both protraction and retraction movements (Fig. 1A). Typically the protraction-retraction sequence was so fast that the two peaks would largely overlap. We ignored double peaks with $<10 \mathrm{~ms}$ interpeak intervals. Thus, each marked peak represented a single whisker movement.

Respiratory behavior was monitored based on temperature changes associated with the expiration of warm air. A fast thermistor (response time $200 \mathrm{~ms}$, Measurement Specialties, Inc.), was placed in front of one nostril and breathing cycles could reliably be measured as positivenegative temperature change pattern during exhale and inhale movements respectively (Fig. 1A). To allow the mouse to accommodate to the head fixation situation we waited $30 \mathrm{~min}$ before starting recordings. During this period the mice calmed down significantly as judged by the much reduced number of attempts to walk or run. Raw respiratory and whisking signals were digitized at $1 \mathrm{kHz}$ and stored to hard drive using a CED power 1401 A/D converter and Spike2 software (both Cambridge Electronic Design). Whisking and breathing activities were recorded simultaneously for at least $2 \mathrm{~min}$ and stored in the same data file.

Data analysis. Stable 120 s recordings of respiratory and whisking behavior were chosen for data analysis. Peaks and troughs in the air temperature recordings corresponded to the ends of expiration and inspiration cycles respectively. Peak and trough times were detected and marked to be used to temporally align the data for the cross-correlation analysis. The times of protraction movements of the whisker were detected as the descending potential crossing a fixed voltage threshold. The minimal interval between successive threshold crossings was set to $40 \mathrm{~ms}$ to prevent marking of retraction movements. Average cross-correlation analyses (Spike2, CED, UK), were performed to investigate the temporal correlation between whisking and respiratory movements across the entire observation period or for brief episodes where the whisking frequency varied. We used the inspiration or expiration times to align the data.

To determine statistical significance of the correlations, we generated surrogate respiratory sequences by randomly shuffling inter-inhale or interexhale intervals and recalculated the correlations between the shuffled respiratory and the unaltered whisking times. This shuffling procedure was repeated 100 times to obtain an estimate of the expected mean and variability (SD) of the correlation based on the null hypothesis that the two behaviors were uncorrelated. Raw correlation results were then statistically evaluated using $Z$-scores based on the expected correlation mean and variability. $Z$-scores were calculated for peak correlation values by subtracting the expected mean and dividing the difference by the expected SD. Z-scores exceeding 3 SDs were considered statistically significant.

To investigate changes in the breathing-whisking correlation over time we calculated time-resolved cross- and auto-correlations of whisking and respiratory behavior within consecutive, nonoverlapping $0.5 \mathrm{~s}$ time windows for temporal delays of $\pm 1 \mathrm{~s}$ at $20 \mathrm{~ms}$ resolution. Correlation coefficients were expressed in color. This analysis was performed using a custom script written in Matlab (MathWorks).

To analyze the correlation between respiratory and whisking movements as a function of whisking rhythm, inter-whisking intervals as they occurred during a 2 min observation period were separated into three groups according to their duration (or corresponding frequency): $1-0.2$ $\mathrm{s}(1-5 \mathrm{~Hz}), 0.2-0.1 \mathrm{~s}(5-10 \mathrm{~Hz})$, and $<0.1 \mathrm{~s}(>10 \mathrm{~Hz})$. The start of each interval was marked as shown below in Figure $4 A$ and used to temporally align the data to calculate cross-correlations for each particular interval or frequency range. Correlation amplitude was expressed as $Z$-scores relative to an expected correlation assuming independence of breathing and whisking. The expected correlation mean and SD under the null hypothesis of temporal independence was calculated by repeatedly $(100 \times)$ shifting the respiratory data in time relative to whisking event train, calculating the cross-correlation for each shift and finally calculating the bin-wise mean and SD of all 100 correlations. Statistical comparisons of $Z$-scores were made using Two Way ANOVA with repeated measures, followed by the Student-Newman-Keuls post hoc test. Statistical analysis was performed using the SigmaStat 3.5 software (Systat). All values are expressed as means $\pm \mathrm{SD}$.

\section{Results}

\section{Whisking and breathing behaviors}

Whisking and breathing activities were recorded simultaneously in 12 adult $\mathrm{B} 6$ mice. Figure $1 \mathrm{~A}$ shows raw data examples of the voltage fluctuations generated by a single whisker passing through the light-beam sensor. The temperature trace representing expiration and inspiration can be seen as increases and decreases in temperature respectively. Autocorrelation histograms of whisking behavior (Fig. $1 B$, left) revealed a single prominent peak at $\sim 100 \mathrm{~ms}$ preceding an elevated plateau, indicative of a preferred frequency of $\sim 10 \mathrm{~Hz}$ embedded in otherwise variable whisking rhythms with an average frequency of $6.12 \pm 1.46 \mathrm{~Hz}$ (mean $\pm \mathrm{SD}$ ). Respiration, by contrast was highly rhythmic at an average frequency of $3.46 \pm 0.37 \mathrm{~Hz}$ (mean $\pm \mathrm{SD}$ ), reflected in the multiple equidistant peaks in the autocorrelation histogram (Fig. $1 \mathrm{~B}$, right). However, while centered around a preferred or resting frequency the respiratory rhythm could vary between $\sim 2$ and $10 \mathrm{~Hz}$. Figure $2 A, B$ shows an example of whisking and respiratory activity as in Figure 1, with an additional trace (bottom) representing the respiratory frequency averaged over $\pm 1 \mathrm{~s}$ for each expiratory event. Sniffing, marked with horizontal bars above the respiratory trace in Figure $2 A$, is typically associated with respiration frequencies $>5 \mathrm{~Hz}$ (Macrides et al., 1982; Uchida and Mainen, 2003; Kepecs et al., 2007; Wesson et al., 2009). In the data example shown in Figure $2 B$ respiratory rate increases from 4 to $6 \mathrm{~Hz}$ for several seconds. Whisking occurs before and after, but not during the period of high-frequency respiration.

Using the data from all 12 mice we marked the end-ofexpiration cycles and whisking events to determine what proportion of respiratory behavior occurred at frequencies $>5 \mathrm{~Hz}$ (i.e., sniffing, Fig. $2 A, B)$ and what proportion of whisking events occurred during high-frequency $(>5 \mathrm{~Hz})$ respiration or sniffing. Figure $2 C$ shows the frequency distribution of the $1 /$ interexpiratory intervals (expressed in percent) and Figure $2 D$ shows the frequency distribution of whisking events (in percent) vs respiratory rate. Respiratory intervals $>5 \mathrm{~Hz}$ accounted for $14.7 \pm$ $3 \%$ of respiratory intervals. Whisking events that occurred during high-frequency respiration accounted for $13.2 \pm 3.2 \%$ of whisker strokes.

\section{Temporal correlation of whisking with breathing}

Cross-correlation histograms of whisking and breathing behavior were calculated for whisking times with end-of-inspiration 


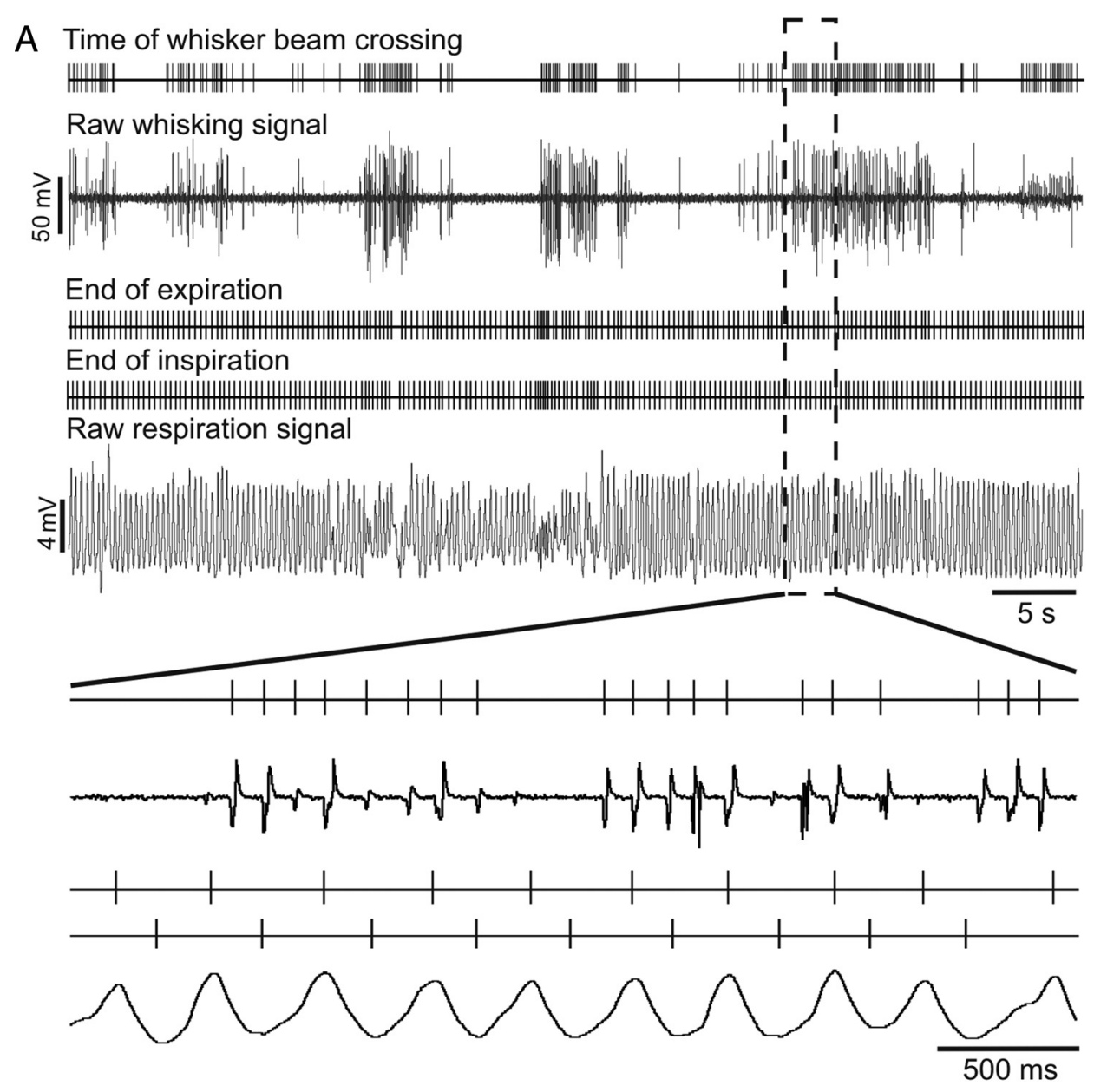

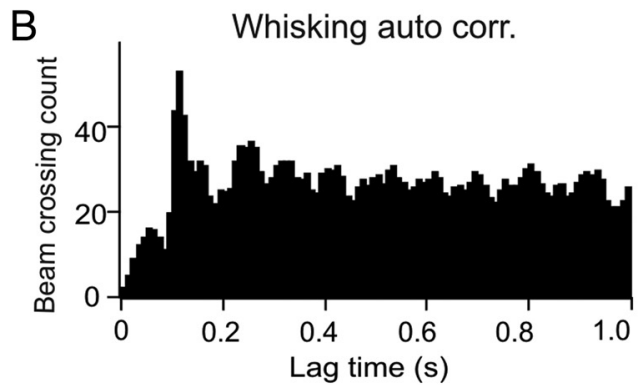

C Whisking-inspiration cross corr.

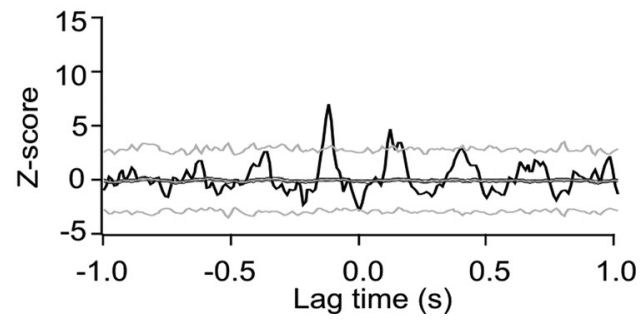

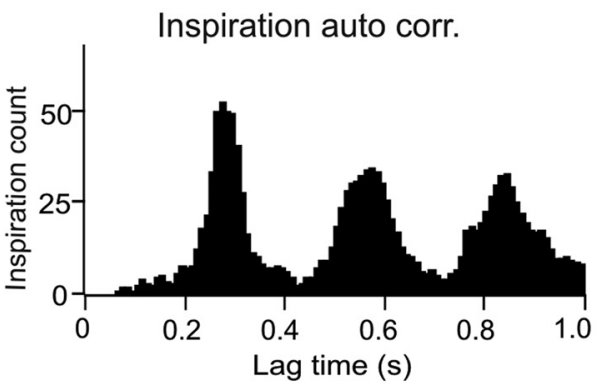

Whisking-expiration cross corr.

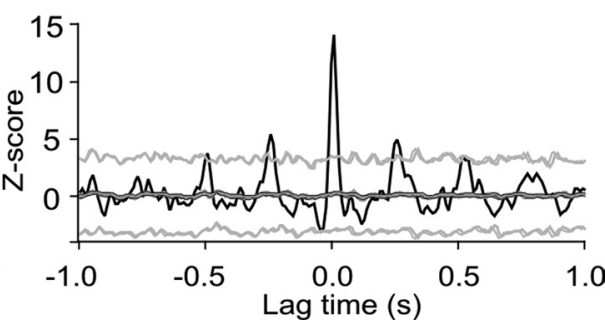

Figure 1. Examples of raw signals and placement of temporal markers for whisking and respiratory behavior and cross-correlation results for whisking and breathing behaviors. $\boldsymbol{A}$, Raw data examples of simultaneously recorded light-beam break and thermistor voltage signals to detect whisker and respiratory movements. Above each raw signal trace are vertical lines marking the times of whisker movements or inspiration and expiration cycles. Negative voltage deflections in the raw whisking signal correspond to the time of whisker blocking the light beam. Peaks and troughs in the raw respiratory signal correspond to the end-of-expiration and end-of-inspiration times respectively. Raw signals and time markers are shown on an expanded time scale below. $\boldsymbol{B}$, Autocorrelation analysis of whisking and respiratory (expiration) behavior. Whisking behavior shows a preferred inter-whisk interval at $\sim 100 \mathrm{~ms}$ ( $10 \mathrm{~Hz}$ ). The autocorrelation of exhale movements shows three clear peaks at multiples of $\sim 310 \mathrm{~ms}$, indicative of a relatively steady $\sim 3.2 \mathrm{~Hz}$ respiratory rhythm. C, Example of a rhythmic correlation between whisking and inspiration (left) and whisking and expiration (right). Black lines represent the raw cross-correlation histograms. Dark gray and light gray lines represent the mean \pm 3 SDs of the expected (shuffled) correlations. Thin black lines above and below the dark gray line represent the SEM. The $y$-axes show Z-scores and the $x$-axes, time delay relative to whisking time. 

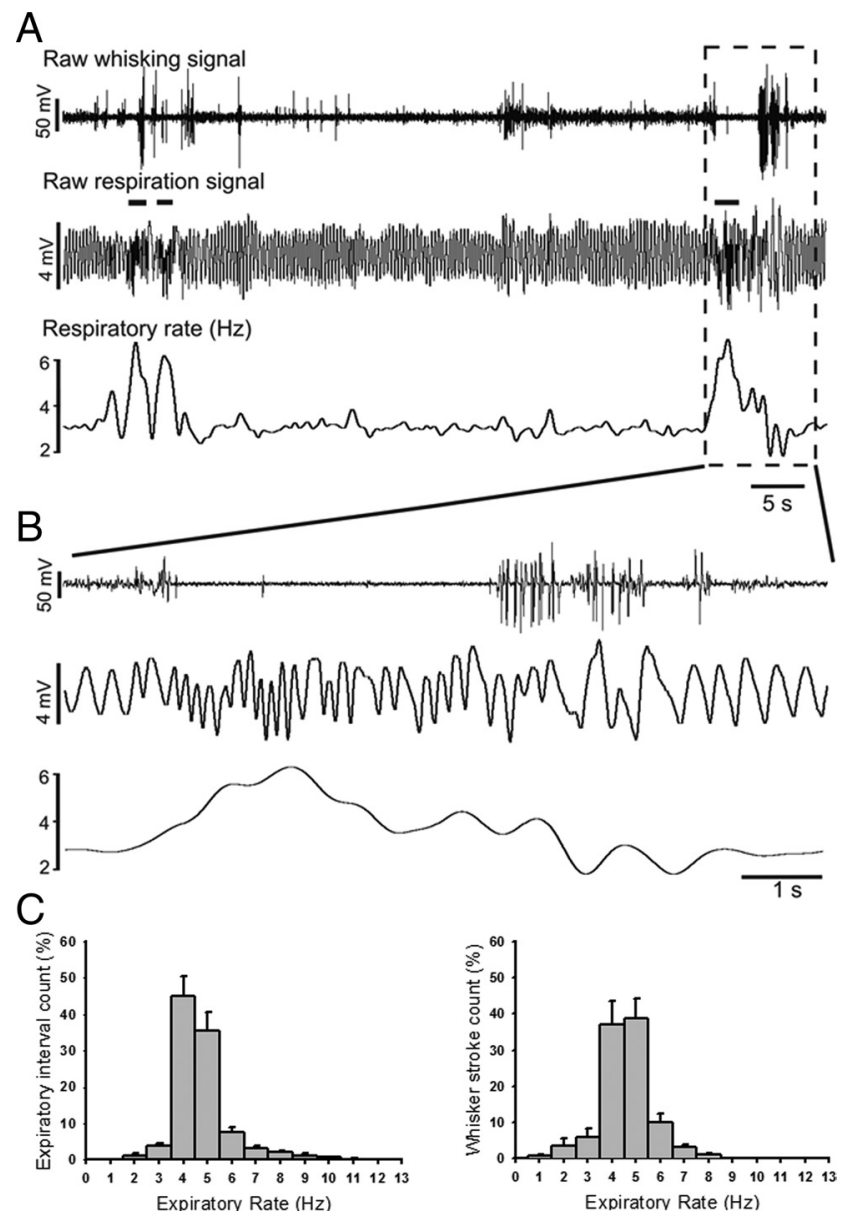

Figure 2. Distribution of whisking events relative to respiratory frequency. $\boldsymbol{A}$, Raw data examples of simultaneously recorded whisking and respiratory behavior during transitions of respiratory rhythm from slow $(<5 \mathrm{~Hz})$ to fast $(<5 \mathrm{~Hz})$ and back. Bottom trace represents the respiratory frequency averaged across inter-expiratory intervals within a $2 \mathrm{~s}$ sliding window. Horizontal bars above the middle trace mark periods of high-frequency respiration, i.e., sniffing. $\boldsymbol{B}$, Expansion of the framed traces in $\boldsymbol{A}$ showing the temporal relationship between highfrequency breathing and large-amplitude whisker movements on an larger time scale. $\boldsymbol{C}$, Left, Frequency histogram (expressed in percent) of inter-expiratory intervals. Right, Frequency histogram (expressed in percent) of whisker strokes during different states of respiratory frequencies. Both histograms show mean values across 5 mice. Error bars represent SEM.

and end-of-expiration times (Fig. 1C). Measurements of whisking and respiratory behavior were performed twice on two consecutive days. The correlation results revealed differences between mice but also suggested that correlation characteristics were stable for individual mice. Eight of 12 mice (66\%) had significant correlations (peak correlation $Z$-scores $\geq 3$ ) between whisking and end-of-inspiration times. Eleven of 12 mice (91\%) had significant correlations between whisking and end-ofexpiration times (example histograms shown in Fig. 1C).

\section{Time-resolved of cross-correlation between whisking and breathing}

Respiratory and whisking frequencies vary over time. We asked whether the correlation between the two behaviors also varied. To this end we performed a time resolved cross-correlation analysis of whisking and breathing times (end-of-expiration). Figure $3 A$ shows an example result of a time resolved correlation analysis over a 2 min recording period. The correlation patterns between whisking and breathing changed over time as a function of whisking frequency. In the color coded time resolved correlation plot
(Fig. 3A) epochs of rhythmic breathing-whisking correlation have a red band at zero delay, with multiple equidistant side bands at delays corresponding to $\sim 4 \mathrm{~Hz}$. The three adjacent time intervals marked by dashed lines show (from left to right) strong, weak and intermediate correlations between breathing and whisking. Across all three intervals the respiratory rhythm remained stable (Fig. 3B,C) but the whisking rhythm changed from $\sim 5 \mathrm{~Hz}$ to $>10 \mathrm{~Hz}$ and back down to an intermediate average. The three histograms at the bottom (Fig. 3D) show the time resolved correlation results averaged across each interval: even though the respiration frequency remained constant, the correlation between whisking and expiration varies with frequency of whisking.

\section{Breathing-whisking correlation for different whisking frequencies}

To quantify the relationship between breathing-whisking correlation strength and whisking frequency, inter-whisking intervals were separated into 3 different frequency groups $(0-5 \mathrm{~Hz}, 5-10$ $\mathrm{Hz}$ and $>10 \mathrm{~Hz}$ ) as illustrated in Figure $4 A$. Breathing-whisking cross-correlation analysis was performed for each of the three whisking frequency ranges by using only whisking events to temporally align the data that preceded intervals of the appropriate frequency range. An example whisking-expiration correlation result for all whisking events and the three frequency ranges is shown in Figure $4 B$. For statistical comparison of correlation strengths $Z$-scores of peak correlation values were determined for each of the four cross-correlations for all 12 mice (Fig. 4C). A two-way ANOVA analysis revealed a significant interaction effect $\left(F_{(3,33)}=3.048 ; p=0.04\right)$ between the group factor (two groups levels: inspiration vs expiration) and whisking frequency factor (4 levels: all, $1-5 \mathrm{~Hz}, 5-10 \mathrm{~Hz}$ and $>10 \mathrm{~Hz}$ ), and a significant overall group effect $\left(F_{(1,33)}=6.14 ; p<0.031\right)$ in $z$ scores. The $Z$-scores for whisking-expiration were higher than whisking-inspiration across all whisking frequencies $(p=0.003)$. When considering individual frequency ranges however, differences were only significant for whisking frequency between 1 and $5 \mathrm{~Hz}$ group $(p=$ $0.019)$ but not for whisking frequencies between 5 and $10 \mathrm{~Hz}$ $(p=0.39)$ or $>10 \mathrm{~Hz}(p=0.16)$. For both inspiration and expiration cycles the correlation with whisking was significantly stronger for low-frequency whisking $(1-5 \mathrm{~Hz})$ than for faster whisking $(>5 \mathrm{~Hz})($ all $p$ values $<0.01)($ Fig. $4 C)$.

\section{Discussion}

Exploratory behavior in mice and rats is characterized by continuous gathering of olfactory and tactile information through fast rhythmic respiratory movements (sniffing) and rhythmic movements of the mystacial vibrissae (whisking), respectively. Because breathing and whisking have overlapping frequency ranges and are performed during the same behavioral context, it has been suggested that the two movements are coordinated with each other. Here we addressed this question in awake, head fixed mice using correlation analysis of the time series of spontaneous whisker and respiratory movements. We show that whisking and breathing movements are correlated when the whisking frequency is low $(<5 \mathrm{~Hz})$. During high-frequency whisking the two movements were uncorrelated. Only a small fraction of whisker movements $(\sim 13 \%)$ occurred during high-frequency rhythmic respiration. Thus, high-frequency sniffing-a behavior fundamental to olfactory processing, and whisking, a behavior fundamental to tactile processing, were mostly nonoverlapping and 

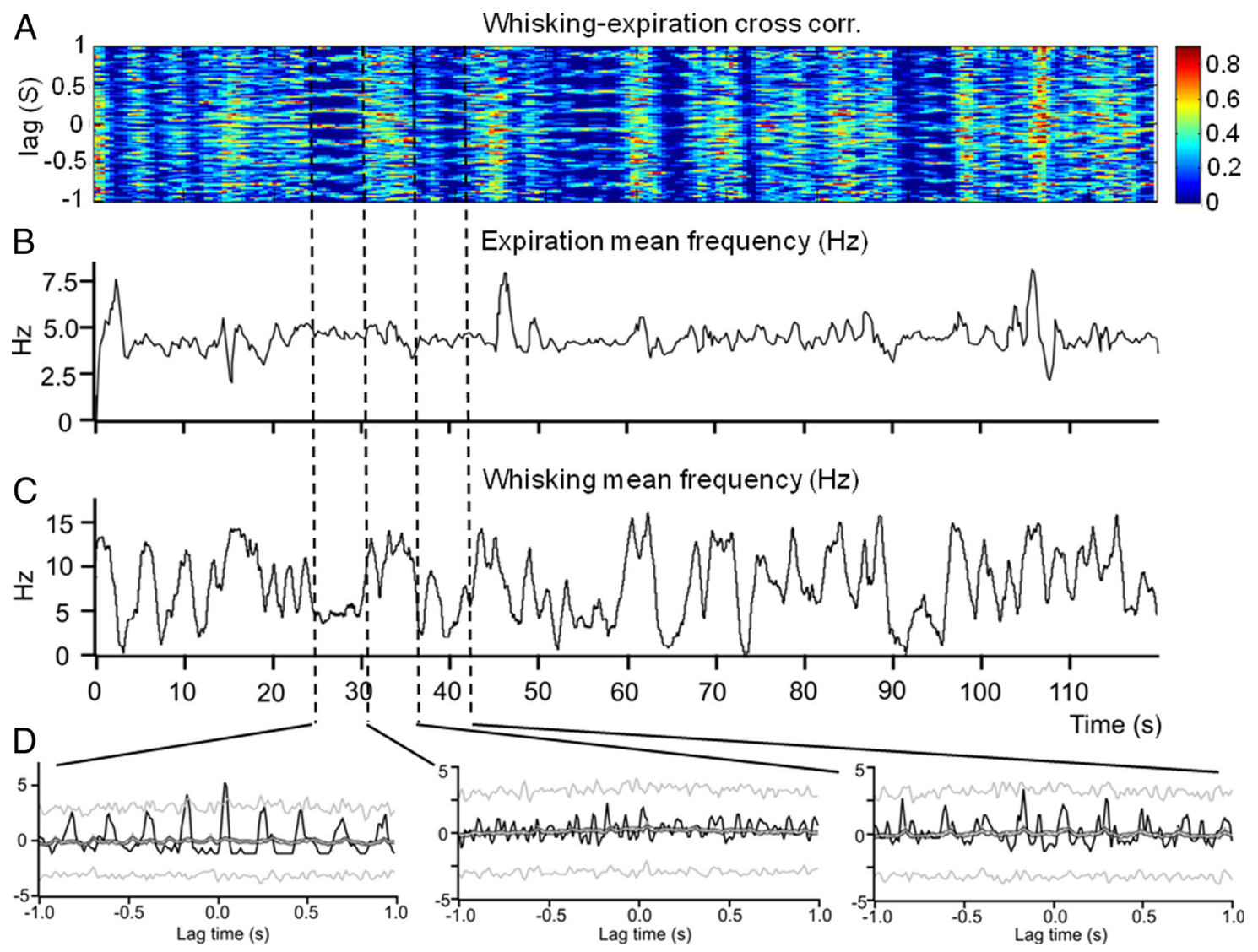

Figure 3. Time resolved correlation analysis reveals dynamic changes in breathing-whisking correlation. $\boldsymbol{A}$, Time-resolved cross-correlation analysis of respiration (expiration) and whisking behavior. $\boldsymbol{B}$, Expiration frequency over time. $\boldsymbol{C}$, Whisking mean frequency over time. $\boldsymbol{D}$, Three cross-correlation histograms calculated during periods of whisker movements at slow ( $\sim 5 \mathrm{~Hz}$, left), high $(>10 \mathrm{~Hz}$, middle) and intermediate (right) frequencies.

separate. This separation could serve to maximize sensory acuity by focusing attention on one modality and avoiding cross modal interference (Driver and Spence, 1998; Koelewijn et al., 2010).

Behavioral modulation of whisking and sniffing oscillations The frequencies of sniffing and whisking vary with behavior. Behavior-dependent variability of whisking frequency has been observed in various behavioral contexts such as exploration, gap crossing, when making contact with objects (Semba and Komisaruk, 1984; Carvell and Simons, 1990; Sachdev et al., 2002; Berg and Kleinfeld, 2003; Mitchinson et al., 2007; Ritt et al., 2008; Grant et al., 2009; Zuo et al., 2011) or when their whiskers have been manipulated during development (Carvell and Simons, 1996). High speed video observations in freely moving mice revealed a negative correlation between peak whisking frequency and amplitude (Voigts et al., 2008) with small amplitude whisker movements $\left(<20^{\circ}\right)$ reaching peak frequencies of up to $30 \mathrm{~Hz}$. Peak frequencies of large-amplitude $\left(>50^{\circ}\right)$ whisker movements, however, were mostly distributed between 10 and $15 \mathrm{~Hz}$. Only large-amplitude whisker movements were captured in the current study and the peak frequency values found by Voigts et al. (2008) correspond well with our findings. The average frequency of high amplitude movements in our hands was $6.12 \mathrm{~Hz}$. Voigts et al. (2008) do not report an average frequency value for largeamplitude movements alone, which makes a direct comparison difficult. Though a lot is known about whisking, whether whisking frequency changes in some defined manner over the course of a tasks is still not well established. By contrast, changes in respiration and sniffing frequency are well documented: respiration frequency typically varies around $2-4 \mathrm{~Hz}$ and can switch to a theta dominated active sniffing frequency of $6-9 \mathrm{~Hz}$ or $9-12 \mathrm{~Hz}$ before or during odor sampling (Adrian, 1951; Kepecs et al., 2007). Sniffing is not fixed in frequency either, it is dynamic, and varies with behavioral context (Welker, 1964; Macrides et al., 1982; Wesson et al., 2008). Together these previous studies demonstrate that frequencies of whisking and sniffing are highly dynamic. Our results suggest that the interaction between these behaviors might be dynamic, breaking down during tactile or olfactory sampling but returning when both breathing and whisking frequencies are slow. It is possible that the head fixation paradigm used here influenced the interaction between breathing and whisking compared with exploratory behavior in a freely moving mouse. Observations in freely moving mice will be necessary to address this question.

A potential problem with light-beam whisker detection is the need for trimming of all whiskers but one, because the altered sensory feedback could alter whisking behavior. However, results from high speed video tracking of whisker movements in freely moving mice suggests that trimming whiskers does not alter inair whisking behavior (Voigts et al., 2008; Zuo et al., 2011). Similarly, complete sensory deafferentation of the whisker pads in rats does not alter in-air whisking behavior (Gao et al., 2001).

Thus far the correlation between breathing and whisking has not been systematically investigated in rats. Whisking behaviors in rats and mice have specific differences including a lower average whisking frequency in rats (Gao et al., 2001; Sachdev et al., 2002; Zuo et al., 2011), which might result in differences in breathing-whisking correlations between rats and mice (De- 
A

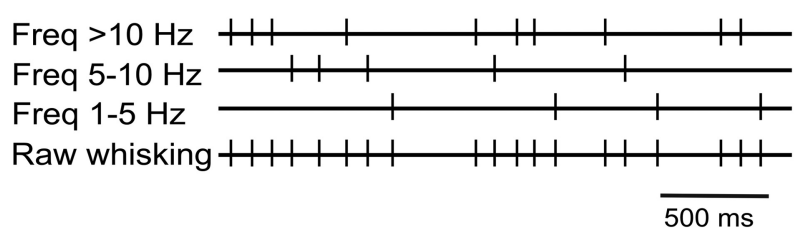

C

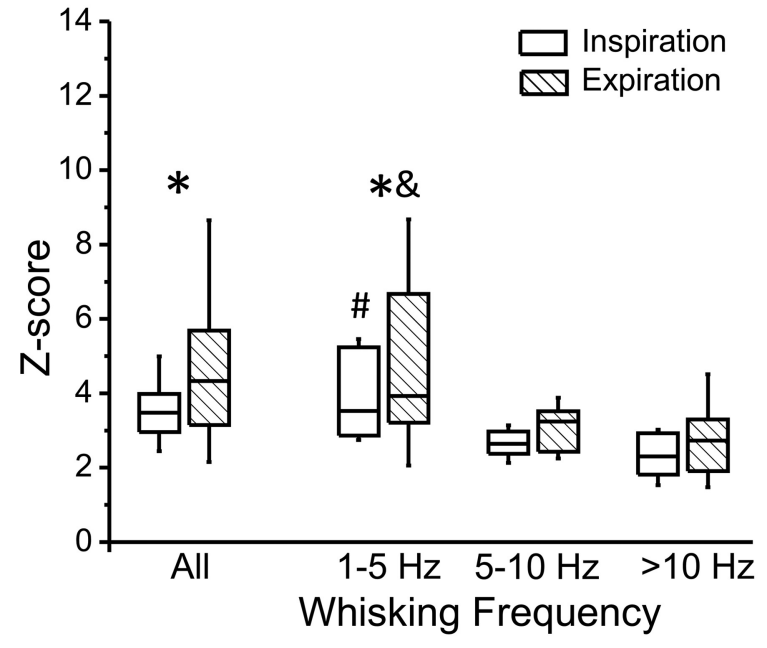

B Whisking-expiration cross corr
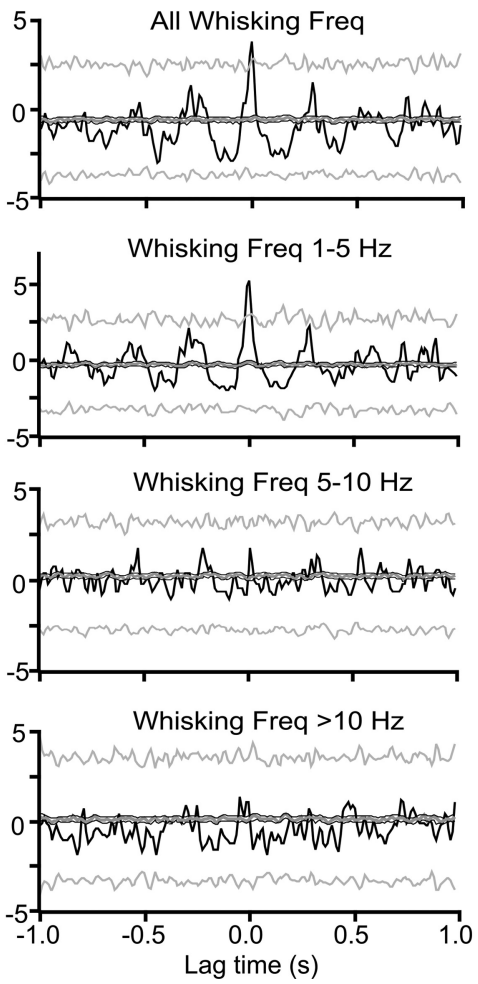

Figure 4. Analysis of whisking-breathing correlations as a function of whisking frequency. $A$, Illustration of the separation of whisking events into 3 frequency groups. Events were assigned to a frequency group based on the duration of the following interval. $\boldsymbol{B}$, (ross-correlation analyses of breathing and whisking behaviors were performed for all whisking events (top) and for inter-whisk intervals of different durations corresponding to the frequency groups indicated above each histogram. C, Average Z-scores of cross-correlation peak values for whisking and respiration separated by whisking frequency. ${ }^{*}$ Significant differences in whisking-breathing correlation strength between inspiration and expiration $(p<0.02)$; ${ }^{*}$ significant differences in whisking-breathing inspiration correlation compared with 5-10 and $>10 \mathrm{~Hz}$ groups $(p<0.01)$; ${ }^{\&}$ significant differences in whisking-breathing expiration correlation compared with $5-10$ and $>10 \mathrm{~Hz}$ groups $(p<0.01)$.

schenes et al., 2011). Our data suggest a temporal separation of olfactory and tactile sensory sampling in mice. Whether this is a general principle valid across species remains to be shown.

The neuronal mechanisms that could potentially link respiration to whisking are not known. The respiratory rhythm is controlled by a well known brainstem circuits (Smith et al., 2009). Whether sniffing is controlled by the same centers in not known (Mainland and Sobel, 2006). Rhythmic whisker movements are controlled by brainstem CGP that includes the facial motor neurons (Cramer et al., 2007). Whether the brainstem circuits for respiratory and whisking rhythms are directly or indirectly connected is unknown.

In summary, our data show that breathing and whisking movements are coordinated in a context-dependent manner with sniffing and high-frequency whisking movements separated in time. It remains to be shown whether the synchronization or desynchronization of whisking and breathing movements serve a behavioral purpose, such as the temporal segmentation of multimodal sensory inputs, and what the underlying neuronal control mechanisms might be.

\section{References}

Adrian ED (1951) The role of air movement in olfactory stimulation. J Physiol 114:4-5p.

Berg RW, Kleinfeld D (2003) Rhythmic whisking by rat: retraction as well as protraction of the vibrissae is under active muscular control. J Neurophysiol 89:104-117.

Bryant JL, Roy S, Heck DH (2009) A technique for stereotaxic recordings of neuronal activity in awake, head-restrained mice. J Neurosci Methods 178:75-79.
Carvell GE, Simons DJ (1990) Biometric analyses of vibrissal tactile discrimination in the rat. J Neurosci 10:2638-2648.

Carvell GE, Simons DJ (1996) Abnormal tactile experience early in life disrupts active touch. J Neurosci 16:2750-2757.

Cramer NP, Li Y, Keller A (2007) The whisking rhythm generator: a novel mammalian network for the generation of movement. J Neurophysiol 97:2148-2158.

Deschenes M, Demers M, Moore JD, Kleinfeld D (2011) Common rhythmic pattern generators drive both whisking and breathing in rats. Soc Neurosci Abstr 37:76.09.

Driver J, Spence C (1998) Crossmodal attention. Curr Opin Neurobiol 8:245-253.

Gao P, Bermejo R, Zeigler HP (2001) Whisker deafferentation and rodent whisking patterns: behavioral evidence for a central pattern generator. J Neurosci 21:5374-5380.

Grant RA, Mitchinson B, Fox CW, Prescott TJ (2009) Active touch sensing in the rat: anticipatory and regulatory control of whisker movements during surface exploration. J Neurophysiol 101:862-874.

Hutson KA, Masterton RB (1986) The sensory contribution of a single vibrissa's cortical barrel. J Neurophysiol 56:1196-1223.

Kepecs A, Uchida N, Mainen ZF (2007) Rapid and precise control of sniffing during olfactory discrimination in rats. J Neurophysiol 98:205-213.

Koelewijn T, Bronkhorst A, Theeuwes J (2010) Attention and the multiple stages of multisensory integration: a review of audiovisual studies. Acta Psychol (Amst) 134:372-384.

Macrides F, Eichenbaum HB, Forbes WB (1982) Temporal relationship between sniffing and the limbic theta rhythm during odor discrimination reversal learning. J Neurosci 2:1705-1717.

Mainland J, Sobel N (2006) The sniff is part of the olfactory percept. Chem Sens 31:181-196.

Mitchinson B, Martin CJ, Grant RA, Prescott TJ (2007) Feedback control in 
active sensing: rat exploratory whisking is modulated by environmental contact. Proc Biol Sci 274:1035-1041.

Ritt JT, Andermann ML, Moore CI (2008) Embodied information processing: vibrissa mechanics and texture features shape micromotions in actively sensing rats. Neuron 57:599-613.

Sachdev RN, Sato T, Ebner FF (2002) Divergent movement of adjacent whiskers. J Neurophysiol 87:1440-1448.

Semba K, Komisaruk BR (1984) Neural substrates of two different rhythmical vibrissal movements in the rat. Neuroscience 12:761-774.

Smith JC, Abdala AP, Rybak IA, Paton JF (2009) Structural and functional architecture of respiratory networks in the mammalian brainstem. Philos Trans R Soc Lond B Biol Sci 364:2577-2587.

Uchida N, Mainen ZF (2003) Speed and accuracy of olfactory discrimination in the rat. Nat Neurosci 6:1224-1229.
Voigts J, Sakmann B, Celikel T (2008) Unsupervised whisker tracking in unrestrained behaving animals. J Neurophysiol 100:504-515.

Welker WI (1964) Analysis of sniffing of the albino rat. Behaviour 22:223-243.

Wesson DW, Donahou TN, Johnson MO, Wachowiak M (2008) Sniffing behavior of mice during performance in odor-guided tasks. Chem Sens 33:581-596.

Wesson DW, Verhagen JV, Wachowiak M (2009) Why sniff fast? The relationship between sniff frequency, odor discrimination, and receptor neuron activation in the rat. J Neurophysiol 101:10891102.

Zuo Y, Perkon I, Diamond ME (2011) Whisking and whisker kinematics during a texture classification task. Philos Trans R Soc Lond B Biol Sci $366: 3058-3069$ 